\title{
Executive Functions in Preschool Children with Aggressive Behavior: Impairments in Inhibitory Control
}

\author{
Maartje A. J. Raaijmakers • Diana P. Smidts • \\ Joseph A. Sergeant • Gerard H. Maassen • \\ Jocelyne A. Posthumus • Herman van Engeland • \\ Walter Matthys
}

Published online: 25 April 2008

(C) The Author(s) 2008

\begin{abstract}
The question whether executive function (EF) deficits in children are associated with conduct problems remains controversial. Although the origins of aggressive behavior are to be found in early childhood, findings from EF studies in preschool children with aggressive behavior are inconsistent. The current study aimed to investigate whether preschool children with aggressive behavior show impairments in EF. From a population-based sample, 82 preschool children who were showing aggressive behavior as indicated by scores at or above the 93rd percentile on the Aggressive Behavior Scale of the CBCL 1 1/2-5 were selected. These children with aggressive behavior were matched on IQ to a group of typically developing control children $(N=99)$. Six neuropsychological tasks were administered to assess set shifting, inhibition, working memory and verbal fluency. A factor analysis was conducted which yielded one clear factor: inhibition. Aggressive preschool
\end{abstract}

M. A. J. Raaijmakers · J. A. Posthumus $\cdot$ H. van Engeland ·

W. Matthys

Department of Child- and Adolescent Psychiatry,

Rudolf Magnus Institute for Neuroscience,

University Medical Center Utrecht,

Utrecht, The Netherlands

D. P. Smidts $\cdot$ J. A. Sergeant

Department of Clinical Neuropsychology,

Vrije Universiteit Amsterdam,

Amsterdam, The Netherlands

G. H. Maassen

Department of Methodology and Statistics, University of Utrecht, Utrecht, The Netherlands

M. A. J. Raaijmakers ( $\square)$

Department of Child- and Adolescent Psychiatry A 01.449/A01.468, University Medical Center Utrecht,

P.O. Box 85500,3508 GA Utrecht, The Netherlands

e-mail: M.A.J.Raaijmakers@umcutrecht.nl children showed poorer performance on this inhibition factor than control children and boys performed worse on this factor than girls. This association between aggressive behavior and inhibition deficits was maintained after controlling for attention problems. In addition, gender differences in all EFs measured were found with boys exhibiting more impairment in EF than girls. These findings demonstrate that preschool children with aggressive behavior show impairments in inhibition, irrespective of attention problems.

Keywords Executive functions - Aggressive behavior . Preschoolers

$\begin{array}{ll}\text { Abbreviations } \\ \text { ADHD } & \text { attention deficit hyperactivity disorder } \\ \text { CD } & \text { conduct disorder } \\ \text { DBD } & \text { disruptive behavior disorder } \\ \text { EF } & \text { executive function } \\ \text { ISI } & \text { inter-stimulus interval } \\ \text { ODD } & \text { oppositional defiant disorder }\end{array}$

\section{Introduction}

Executive functioning (EF) refers to a set of higher order cognitive processes, which are involved in the selfregulation of thought, action and emotion (Séguin and Zelazo 2005). EF processes are necessary for adaptive and goal-oriented behavior, and have been associated with the integrity of neural systems in the prefrontal cortex (Fahie and Symons 2003). A number of different neuropsychological concepts are encompassed within EF, including inhibition, attentional control, working memory, cognitive flexibility or set shifting, goal setting and problem solving 
(Senn et al. 2004). Impairments in executive functioning (EF) have been linked to an impulsive behavioral style and the regulation of aggressive behavior (Séguin et al. 1995). Despite the fact that the origins of persistent aggressive behavior are to be found in early childhood (Shaw et al. 2005), only a few studies into EF have been conducted in preschoolers with aggressive behavior or conduct problems (e.g., Hughes et al. 2000; Senn et al. 2004). In this introduction, the relation between aggressive behavior and $\mathrm{EF}$ in elementary school children and adolescents is discussed first. Second, issues regarding the assessment of EF in young children are addressed. Third, several studies of EF in preschoolers who show aggressive behavior are reviewed and, finally, the aim of the study is presented.

\section{Aggressive Behavior and EF}

Aggressive behavior and conduct problems are often defined in different ways, for example in terms of psychiatric disorders, such as disruptive behavior disorders (DBD). The relation between EF and DBD has been studied (e.g., Morgan and Lilienfeld 2000), but to a lesser extent than the relation between $\mathrm{EF}$ and attention deficit hyperactivity disorder (ADHD; Willcutt et al. 2005). Consequently, the association between ADHD and impairments in EF has been clearly established in research (Willcutt et al. 2005). In their review, Pennington and Ozonoff (1996) concluded that impairments in EF found in children with DBD were due to the presence of comorbid ADHD. Since then, this finding has been confirmed in several other studies (e.g., Clark et al. 2000; Nigg et al. 1998; Oosterlaan et al. 2005). However, in several other studies, the relation between aggression and EF impairments was maintained while controlling for ADHD (Déry et al. 1999; Séguin et al. 1999, 2004). Likewise, in a meta-analysis, Oosterlaan et al. (1998) concluded that deficits in response inhibition in children aged 6 to 12 years were not uniquely associated with ADHD, but also with conduct disorder (CD). In addition, children and adolescents with DBD have been found to show a dysfunction on EF tasks in which motivational processes are involved (Blair et al. 2001; Matthys et al. 1998, 2004; Van Goozen et al. 2004). In conclusion, EF impairments are to be expected in children and adolescents who show aggressive behavior disorders, irrespective of ADHD. It is unclear, however, whether this holds true for preschool children.

\section{Assessment of EF in Young Children}

Historically, young children have been assumed to lack executive capacities (Isquith et al. 2005). For this reason, studies into the EF of preschool children are relatively scarce and results are contradictory. Moreover, there is a paucity of measures to assess EF in young children (e.g., Carlson 2005; Espy et al. 2001). Only a few of the tests originally designed for older children, adolescents or adults that were adapted for 4-year-olds are completely developmentally appropriate for preschool children (Gerstadt et al. 1994; Isquith et al. 2005; Kerr and Zelazo 2004; Welsh et al. 1991). Recently, the preschool period has gained attention with respect to the development of EF. During early childhood EF matures substantially, e.g., between 3 and 4 years of age improvements appear in response inhibition and the ability to think flexibly (Espy 1997; Jacques and Zelazo 2001).

However, no consensus exists concerning which EFs can already be distinguished in preschool children. Exploratory and confirmatory factor analyses have often been used to delineate components of $\mathrm{EF}$ in both adults and children (e.g. Hughes 1998; Miyake et al. 2000). Welsh et al. (1991) suggested at least three executive factors: working memory and planning, inhibition of maladaptive prepotent responses, and self-monitoring or attentional flexibility. Senn et al. (2004) identified working memory, inhibition, and flexibility or shifting as latent executive constructs in a sample of preschoolers, which also have been used in previous studies (e.g., Pennington 1997). A recent review on EF in preschool children shows that the EF components found most often were set shifting, working memory and inhibition (Garon et al. 2008). In confirmatory factor analyses, these three EFs were partially independent but still intercorrelated (Letho et al. 2003; Miyake et al. 2000). Therefore, EF can be viewed as a unitary construct with dissociable components in the preschool years (Miyake et al. 2000).

\section{EF in Preschool Children with Aggressive Behavior}

Studies which did investigate EF in young children who show aggressive behavior revealed impairments in inhibition of maladaptive prepotent responses (Hughes et al. 1998), deficits in planning and inhibitory control in a group of hard-to-manage preschoolers (Hughes et al. 2000), and impaired performance on a motor-planning and attention task and on a semantic classification and working memory task in a group of children who met the criteria for oppositional defiant disorder (ODD), with and without ADHD (Speltz et al. 1999). In other studies of EF in preschoolers with aggressive behavior, the role of ADHD was taken into account more specifically. Results of these studies showed poor EF in children with DBD and comorbid ADHD, but the association between DBD and EF deficits did not remain significant, when the effect of ADHD was partialled out. For example, Kalff et al. (2002) 
showed that children with comorbid DBD and ADHD are more impaired in working memory than children with only DBD. Berlin and Bohlin (2002) and Sonuga-Barke et al. (2002) also found a relationship between deficits in inhibition and ADHD. More recently, Thorell and Wåhlstedt (2006) found that poor EF performance on inhibition, working memory and verbal fluency tasks was associated with symptoms of ADHD, but not with ODD symptoms. In that study, gender differences were also examined but no significant differences between boys and girls were found.

\section{This Study}

To sum up, although the association between DBD or aggressive behavior, irrespective of ADHD, and impairments in EF has been established in elementary school children and adolescents, this association is less clear in preschool children. Yet, the question whether EF impairments are related to disruptive behavior in preschool children is important, because of the high risk for antisocial behavior of children who show early onset aggressive behavior (Moffitt 1993). The early detection of EF impairments might shed a light on the role of neuropsychological deficits in the development of behavior disorders. Therefore, the present study aimed to assess EF in a populationbased sample of 4-year-old children who show aggressive behavior. We hypothesized that preschool children with aggressive behavior problems display impairments in $\mathrm{EF}$, compared to control children. Specifically, based on EF literature in school-age and preschool children, inhibition and working memory are expected to be most clearly impaired. Due to inconsistency (Klenberg et al. 2001; Overman 2004) and scarcity of studies into EF and gender differences, no hypotheses were formulated regarding possible gender differences.

A battery of tasks adapted for preschoolers was used to assess inhibition, working memory, set shifting and fluency. To identify reliable constructs of EF in this sample of young children a factor analysis was performed. Due to the lack of consensus about the definition of EF, especially concerning young children, and because relatively new EF tasks were used in this study, this factor analysis was needed to explore the relation between the different EF variables in preschoolers. In addition, we investigated whether potential EF deficits were due to aggression or attention problems, by partialling out the influence of attention problems. Based on studies into EF impairments in school-aged children and adolescents with conduct problems or aggressive behavior, we hypothesized that EF deficits are mainly explained by aggressive behavior, irrespective of attention problems.

\section{Methods}

Participants

Subjects were selected from a population-based sample of Dutch children in the province of Utrecht. Children were acquired by the Office for Screening and Vaccination. All recruited children were born either in 2000 or 2001 and were 4 years old at the time of assessment. The sample of children used in this study was derived from a longitudinal study into the effect of an indicated preventive intervention, the Incredible Years Parent Training Program (WebsterStratton 2001). In this longitudinal study, neuropsychological measures were included as moderators to predict treatment-effect. Children were selected to participate if they scored at or above the 80th percentile of the Aggressive Behavior Scale of the Child Behavior Checklist 1 1/2-5 (CBCL; Achenbach and Rescorla 2000; Dutch version by Verhulst and Van der Ende); 200 children who showed aggressive behavior were recruited. The present study investigates the neuropsychological performance in a subsample of these children cross-sectionally. We have chosen to include children who clearly show a high level of aggressive behavior and to compare these children to a group with very low levels of aggressive behavior. In this manner, the relation between aggression and EF impairments will become more explicit. Children who showed a high level of aggressive behavior, i.e., if the child scored at or above the borderline range (93rd percentile) of the CBCL Aggressive Behavior Scale were selected to be in the group of children with aggressive behavior (AGGR) in this study. The Aggressive Behavior Scale consists of 19 items, e.g. 'is disobedient' and 'punishment does not change his/her behavior' which are rated on a three-point scale by one of the parents. The AGGR group consisted of 82 children, 59 boys $(72 \%)$ and 23 girls $(28 \%)$. The CBCL was also used in the selection of control subjects, referred to as CONTR. This group was recruited by the Office for Screening and Vaccination. For these 99 control children, 64 boys (64.6\%) and 35 girls (35.4\%), selection was based on a score below the 50th percentile on the Aggressive Behavior Scale and the Attention Problems Scale. The Attention Problem Scale of the CBCL consists of five items, e.g. 'cannot concentrate' and 'cannot sit still', which are also rated on a three-point scale by one of the parents.

Characteristics of the AGGR and CONTR group are depicted in Table 1. Groups were matched on IQ. Children with an estimated full scale IQ below 80 were excluded from this study. None of the children used medication at the moment of assessment. With regard to treatment, two children in the AGGR group received psychosocial treatment in the past 3 months. In addition, parents of 14 
Table 1 Sample Characteristics by Group

\begin{tabular}{|c|c|c|c|c|}
\hline \multirow[t]{3}{*}{ Measure } & \multirow{2}{*}{\multicolumn{2}{|c|}{$\begin{array}{l}\text { AGGR } \\
(N=82)\end{array}$}} & \multirow{2}{*}{\multicolumn{2}{|c|}{$\begin{array}{l}\text { CONTR } \\
(N=99)\end{array}$}} \\
\hline & & & & \\
\hline & Mean & SD & Mean & $\mathrm{SD}$ \\
\hline Age* & 50.65 & 3.05 & 52.31 & 2.20 \\
\hline IQ & 108.33 & 10.40 & 110.31 & 6.97 \\
\hline \multicolumn{5}{|l|}{ CBCL $11 / 2-5$} \\
\hline Aggressive behavior* & 24.93 & 3.80 & 3.67 & 2.57 \\
\hline Attention problems* & 4.90 & 2.16 & 0.55 & 0.90 \\
\hline Total problems* & 65.82 & 16.69 & 12.88 & 10.02 \\
\hline
\end{tabular}

Age is depicted in months; CBCL scores are raw scores. ${ }^{*} p<0.01$

children in the AGGR group consulted a youth care center or a child psychologist because of their child's behavior. Parents of the children in both groups were highly educated and did not significantly differ in their educational level. In the AGGR group 2.5\% received primary education, $4.9 \%$ received secondary education, $29.6 \%$ received intermediate vocational education, $38.3 \%$ received higher vocational education and $24.7 \%$ went to university. In the CONTR group none of the parents received primary education, $6.1 \%$ received secondary education, $29.3 \%$ received intermediate vocational education, $30.3 \%$ received higher vocational education and $34.3 \%$ went to university.

\section{Procedure}

All participants were individually assessed twice in their home environment. Both assessment-sessions took approximately $45 \mathrm{~min}$. Across children, two different testsequences were used to minimize the effect of fatigue and inattention on task performance. Tests were administered by trained experimenters using standardized instructions. On each home visit, two experimenters were present: one assessed the child and the other observed the child. Testing began when instructions were fully understood by the child. Children were asked to be accurate and as fast as possible [except on the Digit Span (words) and the OCTC]; they were not informed of their errors. A HP Compaq Business Notebook NX 9110 was used to run the computerized tasks. The child looked at a Philips 15" LCD-monitor and had to push two large buttons which were converted emergency stop switches with an external diameter of 94 mm (MOELLER Safety Products; FAK-R/V/KC11/1Y). Written informed consent was obtained from the parents of the participating children. Parents completed a set of questionnaires and received a financial reward. Children received a small gift for their participation. This study was approved by the Medical Ethical Review Committee of the Utrecht University Medical Centre.

Measures

Wechsler Preschool and Primary Scale of IntelligenceRevised (WPPSI-R)

During the second assessment, the WPPSI-R (Wechsler 1997; Dutch version by Vander Steene and Bos 1997) was administered to the child to measure intelligence. Subtests Picture Completion, Vocabulary, Block Design and Similarities were used to estimate full scale IQ (correlation subtests with full scale $\mathrm{IQ}=0.92$ ), following the guidelines of Sattler (1992).

\section{Go/No Go}

The Go/No go is a well-established measure of inhibitory control with adequate psychometric properties (Casey et al. 1997; Drewe 1975; Picton et al. 2006). In this study an adaptation of the original Go/No go paradigm was used, adjusted to 4-year-old children (Smidts 2003). Children were shown pictures of an elephant (Go-stimulus) or a dog (No go-stimulus) alternately on a monitor. Pictures were presented for 1,500 ms but disappeared when a response was given within this period. Trials were presented with a fixed inter-stimulus-interval (ISI) of 1,500 ms. Inhibition was required in $50 \%$ of the trials, which were presented in a random order. The task commenced with 48 practice trials, followed by 48 trials which measured task performance. During the practice period the experimenter repeated the instructions to ensure that the child understood the task. Duration of the Go/ No go was approximately $5 \mathrm{~min}$. Task performance was measured by the number of correct and incorrect inhibition responses, and the number of nonresponses.

\section{Digit Span (Words)}

This task is an adaptation of the Digit Span (words) subtest of the Wechsler IQ Scale for Children (WISC; Wechsler 1949) and was used to measure verbal working memory (Smidts 2003). The Digit Span shows an adequate level of internal consistency (Elliot 1990). The task required the child to repeat a string of words, which was read aloud. The forward condition started with two-word strings, which the child had to repeat. When the child repeated these words accurately, the strings were elaborated with one word, until a six-word sequence. In each trial, there were two strings of words; at least one of these strings had to be repeated correctly in order to proceed to the next trial. In the backward condition, the child had to repeat the words in 
reversed order. Again, in each sequence one word was added. Scores obtained from this task were the total number of words the child repeated correctly in the forward- and backward condition.

\section{Shape School}

Originally, the Shape School (Espy 1997) is a colorful storybook, used to measure working memory, inhibition and switching processes. Espy et al. (2006) found evidence of good validity and acceptable reliability for this task. In the present study, a computerized modified version of the test was used (Smidts 2003). The task consisted of four conditions. First, the Control Condition in which the child had to push the button of the color of the figure that appeared on the screen (red or yellow). Second, the Inhibition Condition; the child had to respond by pushing the button of the correct color only when the figure looked happy, and to suppress this response when the figure looked sad. In these two conditions, each consisting of 24 practice trials and 24 trials to measure task performance, there was a fixed ISI of 1,500 ms. Figures were presented for 1,500 ms, but disappeared when a button was pushed within this period. In the two following conditions, Switching and Both, the child had to retain and switch between rules. Again, each condition consisted of 24 trials, but these were now presented on the screen for $2,000 \mathrm{~ms}$ with an ISI of 2,000 ms. In the Switching Condition, the child had to respond to the color of the figure, but when the figure wore a hat, the child had to push the button of the contrasting color. In the Both Condition one rule was added; only push a button when the figure looked happy. In this last condition, the child had to inhibit a response when a sad figure was shown and to switch between rules from earlier conditions. Task performance was measured by the number of correct and incorrect responses, the number of correct and incorrect inhibition responses, and the number of nonresponses in each condition.

\section{Verbal Fluency}

The Verbal Fluency task was designed to measure working memory or semantic word fluency (Welsh et al. 1991). This task required the generation of as many words as possible in a specific category within a given time limit. Children were required to generate as much different examples of 'animals' and 'food and drinks' as they knew, within a time limit of $40 \mathrm{~s}$. Two examples of each category were provided in the task instructions. Items named more than once and items from other categories were rated as incorrect. Scores obtained from this task were the total numbers of correct and incorrect examples the child named in each category.
Object Classification Task for Children (OCTC)

The OCTC (Smidts et al. 2004) was based on the Concept Generation Test for Children (Jacobs et al. 2001). The OCTC is used to examine set shifting or cognitive flexibility. This sorting task required the child to group six pictures of planes and cars on common features in three different ways; color (red or yellow), function (plane or car) and size (big or small). The child was required to form two groups and was then asked for a verbal response on the common feature of the pictures, until all cards were sorted according to the groupings mentioned above. Two practice trials were given, to see whether the child was capable of forming two groups according to overall appearance. The OCTC was employed with two different settings, one with six pictures and one with four pictures. There were three conditions with increasing levels of structure; a Free Generation Condition, where the child had to group the pictures without assistance. For each correct sort, the child received three points. When the child was unable to sort the cards correctly, the setting with four cards was used. The three pictures of the planes were removed and replaced by a picture of a small yellow car, which allowed for sorts of color and size only. In both settings, the Identification Condition was next, in which the experimenter grouped the pictures and the child had to identify the sort. In this condition, two points were given for a correct answer. If the child failed this condition, the experimenter went on to the third condition, Explicit Cueing. The child was explicitly told how to group the pictures. One point was awarded for a correct sort and one point was also given for each correct verbal response. The summed total of points was used as an indication of the child's ability to shift between concepts.

\section{Day-Night Task}

The Day-Night task is a well-validated measure of prepotent response inhibition and working memory in young children (Diamond et al. 2002; Gerstadt et al. 1994). The task requires the child to keep two rules in mind and to inhibit an automatic response. In this study, the experimenter showed the child sixteen cards in a fixed order with either a sun with a blue background, or a white moon and stars with a black background. To ensure that the children adopted a set of prepotent responses, a control condition was administered. In the control condition children had to say "day", when the card with the sun was shown, and "night", when the card with the moon was shown. In the experimental condition, the rules were reversed; the child had to say "day", when it saw the card with the moon, and "night" to the card with the sun on it. A practice trial was administered in each condition, with a 
maximum of three trials, in which the child had to respond correctly to two cards. Scores obtained by this task were the total number of correct and incorrect responses, the number of self-corrections.

\section{Results}

\section{Data Analysis}

Missing data were primarily the result of children who failed to understand task instructions or whose assessment could not be completed due to extreme inattentive or noncompliant behavior. A score of 0 was only given, when the child had attempted the task and then failed. Groups were matched on IQ. The AGGR and CONTR group differed significantly on age (see Table 1); therefore age was used as a covariate. First, a factor analysis was performed on data of all children. Second, ANCOVAs were conducted using a factorscore and other task variables, in which both group and gender were used as betweensubject factors. All analyses were performed using SPSS 15.0 (2006).

\section{Factor Analysis}

To explore the relation between EF variables, scores from the neuropsychological tasks were submitted to an exploratory factor analysis. Principal axis factoring (PAF) was performed on ten variables from different neuropsychological tasks. For this factor analysis, one variable of each task was chosen to prevent an artificial clustering of variables from the same task. To investigate impairments in EF, the number of incorrect responses of the child was used as main outcome variable. However, not every EF task used was designed to measure the number of incorrect responses, especially not the manually administered tasks. Therefore, we used the number of incorrect responses the children gave on the computerized tasks and for the manually administered tasks, we employed the number of correct responses. Using the number of correct responses of the computerized tasks yielded a comparable factor solution, but factors could less clearly be distinguished. Therefore, we chose to include the number of correct responses on the manually administered task and the number of incorrect responses on the computerized tasks.

A PAF followed by varimax rotation was performed and three factors with eigenvalues greater than 1 were extracted. This solution accounted for $33.13 \%$ of the variance. Orthogonal and oblique rotation resulted in the same factor solution. Item loadings of 0.40 or higher are depicted in Table 2. The first factor accounted for $16.67 \%$ of the variance, with factor loadings pointing to errors of
Table 2 Item Loadings of the Rotated Factor Matrix

\begin{tabular}{lccc}
\hline Variable & Factor 1 & Factor 2 & Factor 3 \\
\hline SS Inh: incorrect inhibition & 0.783 & & \\
SS Inh: incorrect & 0.558 & & \\
GNG: incorrect inhibition & 0.537 & & \\
SS Both: incorrect inhibition & 0.488 & & \\
SS Both: incorrect & & 0.701 & \\
OCTC correct & & & 0.488 \\
DS correct & & & 0.481 \\
VF correct & & & 0.413 \\
\hline
\end{tabular}

SS Inh shape school inhibition condition, GNG go no go, SS Both shape school both condition, OCTC object classification task for children, $D S$ digit span (words), $V F$ verbal fluency

commission on the inhibition trials in several computerized tasks. Therefore, this factor, measuring impairments in inhibition, was labeled 'Inhibition'. The internal consistency of this factor was 0.69 . The second factor accounted for $8.87 \%$ of the variance and consisted of only one item, measuring both working memory and set shifting. This task variable, the number of incorrect responses of the Shape School Both condition, was analyzed separately. The third factor accounted for $7.66 \%$ of the variance and consisted of variables from three manually administered tasks. The internal consistency of this factor was 0.39 . Factor loadings are depicted in Table 2. Due to the low percentage of explained variance of the factor analysis and the low internal consistency of the third factor, we decided to include only the Inhibition factor in the analysis. All other tasks variables were analyzed separately.

\section{ANCOVAs}

Significant correlations were only found between variables included in the Inhibition factor. Therefore, one-way ANCOVAs with age as a covariate were carried out to compare performance of the AGGR group on the Inhibition factor and the other task variables to the CONTR group (see Table 3). To conduct the ANCOVA on the Inhibition factor, a factorscore was constructed by transforming the inhibition variables from different tasks into standardized scores. This $z$-score transformation enhances the comparability among the variously scored tasks. Next, the Inhibition factorscore was computed by dividing the total standardized score of the four variables by the number of variables included in the factor. Group and gender were entered in the analysis as between-subject-factors. Means, standard deviations and the results of the ANCOVAs of the Inhibition factor and of the other task variables are displayed in Table 3, for group and gender. Effect sizes were also calculated (see Table 3), using Cohen's $d ; 0.2$ 
Table 3 Mean Scores and Standard Deviations by Group and Gender

\begin{tabular}{|c|c|c|c|c|c|c|c|c|c|c|c|c|c|c|}
\hline \multirow[t]{3}{*}{ Measures } & \multicolumn{4}{|l|}{ AGGR } & \multicolumn{4}{|c|}{ CONTR } & \multirow{2}{*}{\multicolumn{3}{|c|}{ Group }} & \multirow{2}{*}{\multicolumn{3}{|c|}{ Gender }} \\
\hline & \multicolumn{2}{|l|}{ Boys } & \multicolumn{2}{|l|}{ Girls } & \multicolumn{2}{|l|}{ Boys } & \multicolumn{2}{|l|}{ Girls } & & & & & & \\
\hline & Mean & $\mathrm{SD}$ & Mean & $\mathrm{SD}$ & Mean & SD & Mean & SD & $F$ & $P$ & $D$ & $F$ & $P$ & $D$ \\
\hline Factorscore Inhibition & 0.34 & 0.93 & -0.09 & 0.71 & -0.85 & 0.52 & -0.36 & 0.29 & 9.90 & 0.00 & 0.57 & 10.31 & 0.00 & 0.57 \\
\hline SS Inh: incorrect & 1.41 & 2.13 & 0.70 & 1.49 & 0.73 & 1.00 & 0.49 & 1.04 & 4.40 & 0.04 & 0.35 & 3.49 & 0.06 & 0.33 \\
\hline SS Inh: incorrect inhibition & 2.25 & 3.43 & 1.39 & 2.86 & 0.95 & 1.67 & 0.46 & 1.25 & 6.50 & 0.01 & 0.48 & 2.92 & 0.09 & 0.31 \\
\hline $\begin{array}{l}\text { SS Both: incorrect } \\
\text { inhibition }\end{array}$ & 2.47 & 3.31 & 0.70 & 1.02 & 1.75 & 3.27 & 0.37 & 0.88 & 2.10 & 0.15 & 0.25 & 11.95 & 0.00 & 0.66 \\
\hline GNG: incorrect inhibition & 3.00 & 3.16 & 2.22 & 3.15 & 1.66 & 2.21 & 0.91 & 0.92 & 7.26 & 0.01 & 0.54 & 3.78 & 0.05 & 0.35 \\
\hline VF correct & 6.58 & 2.62 & 8.04 & 3.61 & 7.27 & 3.32 & 8.54 & 3.44 & 0.33 & 0.57 & 0.23 & 7.80 & 0.01 & 0.43 \\
\hline DS correct & 4.88 & 1.85 & 5.17 & 2.19 & 5.47 & 2.03 & 5.69 & 2.14 & 0.84 & 0.36 & 0.29 & 0.87 & 0.35 & 0.14 \\
\hline DN correct & 10.12 & 4.26 & 11.74 & 3.41 & 11.91 & 4.23 & 12.20 & 3.64 & 2.25 & 0.14 & 0.36 & 2.21 & 0.14 & 0.25 \\
\hline OCTC correct & 6.02 & 1.68 & 6.96 & 1.94 & 6.39 & 1.44 & 6.46 & 1.50 & 0.14 & 0.71 & 0.08 & 3.85 & 0.05 & 0.28 \\
\hline SS Both: incorrect & 3.69 & 2.65 & 2.83 & 2.73 & 3.80 & 2.97 & 3.77 & 3.18 & 0.89 & 0.35 & 0.12 & 0.87 & 0.35 & 0.12 \\
\hline SS Switch: incorrect & 6.76 & 4.58 & 2.39 & 3.19 & 3.80 & 3.65 & 3.49 & 4.04 & 1.53 & 0.22 & 0.44 & 13.18 & 0.00 & 0.53 \\
\hline
\end{tabular}

ANCOVA group $\times$ gender; Age $=$ covariate. The Inhibition factorscore is based on $z$-scores and a high factorscore implicates poor performance; $p$-values are two-tailed

SS Inh shape school inhibition condition, SS Both shape school both condition, $S S S w$ shape school switching condition, GNG go no go, $V F$ verbal fluency, $D S$ digit span (words), $D N$ day night task, OCTC object classification task for children

indicates a small effect, 0.5 a medium effect and 0.8 a large effect size (Cohen 1992).

The factorscore for Inhibition was included in the first ANCOVA. The four variables that were included in the factorscore were examined in subsequent analyses. Effects for both group and gender were found to be significant on the Inhibition factor and showed medium effect sizes. For group, other variables that yielded significant effects were only the variables included in the Inhibition factorscore. Age as a covariate did not affect the results on the Inhibition factor. Moreover, inspection of the data pointed out that the difference in inhibition scores was not affected by the distribution of age over the groups. The group effects on the Inhibition factor, and on variables included in this factor, pointed in the expected direction, with the AGGR group demonstrating more incorrect inhibition-trials than the CONTR group. This implies that the AGGR group was significantly more impaired in inhibition than the CONTR group. In addition, the Pearson product-moment correlation between the Aggressive Behavior score of the CBCL and the Inhibition factor was computed $(r=0.287$, $p=0.00$ ), which implies that a higher aggressive behavior score was associated with more impairments in inhibition. Gender effects were also found on the Inhibition factor, and on the task variables included in this factor, with girls outperforming boys.

Unlike the effects of group, the effects of gender were not limited to tasks measuring inhibition. Significant gender effects were also reported on the Verbal Fluency task and the number of errors in the Shape School Switch condition, with medium effect sizes. Girls performed better than boys on these task variables. In addition, the effect of gender was marginally significant for the OCTC, but the effect size was smaller than for the other significant effects of gender. Again, girls were outperforming boys. Only one significant interaction effect for group and gender was manifested, i.e., the number of errors on the Shape School Switch condition [group $\times$ gender, $F(1,181)=9.91, p=0.00$ ]. Covarying for age did not yield significant effects on any task variable, except for the Digit Span (words) [age, $F(1,181)=5.61, p=$ $0.02]$. No significant results for group or gender were found on this variable.

To control for the influence of Attention Problems, ANCOVAs were also carried out with Attention Problems as an additional covariate. The Inhibition score remained significant for group [group, $F(1,181)=5.27, p=0.02$ ] and gender [gender, $F(1,181)=10.66, p=0.00$ ]. The significant effect of group for the number of errors on the Shape School Inhibition condition was also maintained [group, $F(1,181)=5.95, p=0.02]$. The effect of group for the variable Shape School Inhibition incorrect responses disappeared and the effect of the Go/No go did not yield significant results for group anymore.

\section{Discussion}

The aim of the current study was to examine EF in preschool children with aggressive behavior in a populationbased sample. Our findings demonstrated that preschoolers who show primarily aggressive behavior displayed impairments in inhibition. In this group of aggressive preschoolers 
these impairments in inhibition were maintained after controlling for attention problems. In addition, gender differences in EF were found, with boys exhibiting more EF deficits than girls.

We first examined which EF could be distinguished in this non-clinical sample of preschool children by factor analysis. Although the neuropsychological tests in this study were aimed at assessing working memory, inhibition, fluency, and set shifting, the only EF factor, which could clearly be distinguished was Inhibition. The other EF could not be distinguished as separate constructs. This implies that the differentiation of EF at this young age remains a complicated issue (Senn et al. 2004). EF is still maturing in the preschool period and will to develop into more specific functions. Considering the complexity of the construct of EF in the preschool years, the finding of inhibition as the only EF factor in the present study indicates that inhibition is a robust concept of EF at four years of age.

An explanation for identifying only inhibition as an EF factor is that inhibition is one of the first EF to emerge (Barkley 1997; Brocki and Bohlin 2004). The ability to inhibit prepotent responses generally increases significantly over the preschool period, which is necessary for the exertion of control over one's behavior (Espy 1997). Therefore, it appears to be a prominent feature in this period of rapid cognitive development, whereas other EF cannot yet be clearly detected at this young age and develop over time (Korkman et al. 2001). Aggressive behavior was only found to be associated with impairments in inhibition and not in other EF. In the meta-analysis of Oosterlaan et al. (1998) and in the study of Hughes et al. (1998) deficits in inhibition were also related to aggressive behavior problems. Inhibition problems constitute a key characteristic of aggressive behavior problems and are found to be persistent over time (Brophy et al. 2002). Although the relation between inhibition problems and DBD is well established in school-aged children, adolescents and adults (Morgan and Lilienfeld 2000), finding this relation in a sample of young children who show aggressive behavior is important, because it might be that inhibition plays a crucial role in the developmental trajectories of aggression.

In contrast to evidence suggesting that EF deficits in preschoolers with aggressive behavior are mainly explained by symptoms of ADHD (e.g. Sonuga-Barke et al. 2002; Thorell and Wåhlstedt 2006), this study reported results which demonstrated that inhibition deficits were significantly related to aggressive behavior, irrespective of attention problems. Attention problems did not influence the effect of aggression on the Inhibition factor. It should, however, be specified that the level of attention problems was relatively low in the present sample. These findings are consistent with the notion that when problems concerning the inhibition of behavior arise, the risk of the development of aggressive behavior is increased (Kochanska et al. 2000). However, even in young children who primarily show aggressive behavior, attention problems remain important because of deficits in working memory and set shifting.

Gender differences in inhibition, verbal fluency, working memory and set shifting were found irrespective of aggression or attention problems. These findings contrast with studies of Overman (2004) and Thorell and Wåhlstedt (2006), in which no differences in EF of boys and girls at this age were manifested. However, gender differences in effortful control, i.e., the ability to inhibit a dominant response to perform a non-dominant response have often been reported. Kochanska et al. (2000) found gender differences with girls outperforming boys in inhibiting impulsive responding, at ages as young as of 22 and 33 months. Olson et al. (2005) reported a significant effect for gender; girls showed higher levels of effortful control than boys at age three. The more rapid developmental maturation of girls (Keenan and Shaw 1997) might be responsible for their higher level of inhibitory skills and other EF in the preschool period and could explain why preschool boys make more mistakes on EF measures. Especially the relative delay in inhibition of preschool boys makes them more prone to the development of aggressive behavior. In further research it is important to study the inhibitory skills of large samples of boys and girls separately in order to more clearly detect gender differences. We also found a difference between boys and girls in verbal fluency, with girls being more verbally fluent than boys. This is relevant as poor verbal skills compose a risk factor for the development of aggressive behavior problems (Loeber et al. 1998). Children at high risk for DBD are children who experience difficulties regarding both executive and verbal cognitive skills (Moffitt 1993). In addition, socialization practices might contribute to gender differences in inhibition. In general, girls are encouraged to exert more control over their behavior than boys (Keenan and Shaw 1997). As a result, girls learn to show more inhibited behavior than boys from an early age on, which might be reflected in our findings. Finally, the marginally significant effects of gender on other EF might be due to a lack of statistical power, because the number of girls in this sample was relatively small. Therefore, EF such as working memory and set shifting also require to be analyzed separately for boys and girls in future studies.

This study has a number of limitations that need to be considered. First, the majority of the parents of the children in our sample showed a high educational level. Therefore, our findings have limited generalizability to children from less educated parents. Second, we employed extreme inclusion criteria. We compared a group of children who showed a low level of aggressive behavior to a group of children who showed a relatively high level of aggressive behavior; these groups represent the extreme ends of the 
behavioral spectrum. The finding of EF impairments in the aggressive group can only be interpreted within this context. Third, we used an experimental battery of tasks, consisting of six neuropsychological measures. The use of other EF measures might have yielded different EF factors, and might thus have captured other EF deficits present in this group of young aggressive preschoolers. An additional limitation is that the measures used did not assess 'pure' EFs; most EF tasks measured more than one EF. Tapping pure EFs is conceptually not feasible, because almost every task requires a subject to keep rules in mind and thus addresses working memory next to the EF which was aimed to be measured. By conducting a factor analysis, we deducted the common variance between the variables from the measures used, resulting in a latent Inhibition factor that represents a more pure measure of EF. The use of tasks which assess the more motivational aspects of EF are of interest, considering that school-aged children and adolescents with DBD show dysfunctions on these kinds of tasks (Blair et al. 2001; Matthys et al. 1998, 2004; Van Goozen et al. 2004). Future studies are needed to clarify the role of reward and the more affective aspects in the EF of children who show aggressive behavior in this young age group.

The relevance of assessing EF in the preschool years is clearly supported by the current findings. Results of this study show that impairment in inhibition is a correlate of aggressive behavior in preschool children, regardless of attention problems. This study adds to the growing body of literature on the role of neuropsychological deficits in the development of behavioral disorders. Since inhibition deficits may contribute to the development of aggressive behavior and DBD, future research should assess EF in aggressive preschoolers longitudinally to gain insight in the role of EF deficits as precursors or risk factors for the development and persistence of DBD.

Acknowledgements We are grateful to the parents and children who participated in this study.

The original research project was funded by ZonMw Prevention (\#2620.0001).

Open Access This article is distributed under the terms of the Creative Commons Attribution Noncommercial License which permits any noncommercial use, distribution, and reproduction in any medium, provided the original author(s) and source are credited.

\section{References}

Achenbach, T. M., \& Rescorla, L. A. (2000). Manual for the ASEBA preschool forms and profiles. Burlington, VT: University of Vermont, Research Center for Children, Youth \& Families.

Barkley, R. A. (1997). Behavioral inhibition, sustained attention, and executive functions: Constructing a unifying theory of ADHD. Psychological Bulletin, 121, 65-94.
Berlin, L., \& Bohlin, G. (2002). Response inhibition, hyperactivity, and conduct problems among preschool children. Journal of Clinical Child Psychology, 31, 242-251.

Blair, R. J. R., Colledge, E., \& Mitchell, D. G. V. (2001). Somatic markers and response reversal: Is there orbitofrontal cortex dysfunction in boys with psychopathic tendencies? Journal of Abnormal Child Psychology, 29, 499-511.

Brocki, K. C., \& Bohlin, G. (2004). Executive functions in children aged 6 to 13: A dimensional and developmental study. Developmental Neuropsychology, 26, 571-593.

Brophy, M., Taylor, E., \& Hughes, C. (2002). To go or not to go: Inhibitory control in hard-to-manage children. Infant and Child Development, 11, 125-140.

Carlson, S. M. (2005). Developmentally sensitive measures of executive function in preschool children. Developmental Neuropsychology, 28, 595-616.

Casey, B. J., Trainor, R. J., Orendi, J. L., Schubert, A. B., Nystrom, L. E., Giedd, J. N., et al. (1997). A developmental functional MRI-study of prefrontal activation during performance of a go-no-go task. Journal of Cognitive Neuroscience, 9, 835-847.

Clark, C., Prior, M., \& Kinsella, G. J. (2000). Do executive function deficits differentiate between adolescents with ADHD and oppositional defiant/conduct disorder? A neuropsychological study using the six elements test and Hayling sentence completion test. Journal of Abnormal Child Psychology, 28, 403-414.

Cohen, J. (1992). A power primer. Psychological Bulletin, 112, 155-159.

Déry, M., Toupin, J., Pauzé, R., Mercier, H., \& Fortin, L. (1999). Neuropsychological characteristics of adolescents with conduct disorder: Association with attention deficit hyperactivity and aggression. Journal of Abnormal Child Psychology, 27, 225-236.

Diamond, A., Kirkham, N., \& Amso, D. (2002). Conditions under which young children can hold two rules in mind and inhibit a prepotent response. Developmental Psychology, 38, 352-362.

Drewe, E. (1975). Go-no go learning after frontal lobe lesions in humans. Cortex, 11, 8-16.

Elliot, C. D. (1990). Differential ability scales. San Antonio, TX: The Psychological Corporation.

Espy, K. A. (1997). The Shape School: Assessing executive function in preschool children. Developmental Neuropsychology, 13, 495-499.

Espy, K. A., Bull, R., Martin, J., \& Stroup, W. (2006). Measuring the development of executive control with the Shape School. Psychological Assessment, 18, 373-381.

Espy, K. A., Kaufmann, P. M., Glisky, M. L., \& McDiarmid, M. D. (2001). New procedures to assess executive functions in preschool children. Clinical Neuropsychologist, 15, 46-58.

Fahie, C. M., \& Symons, D. K. (2003). Executive functioning and theory of mind in children clinically referred for attention and behavior problems. Journal of Applied Developmental Psychology, 24, 51-73.

Garon, N., Bryson, S. E., \& Smith, I. M. (2008). Executive Function in preschoolers: A review using an integrative framework. Psychological Bulletin, 134, 31-60.

Gerstadt, C. L., Hong, Y. J., \& Diamond, A. (1994). The relationship between cognition and action: Performance of children $31 / 2$ 7 years old on Stroop-like day-night test. Cognition, 53, 129-153.

Hughes, C. (1998). Executive function in preschoolers: Links with theory of mind and verbal ability. British Journal of Developmental Psychology, 16, 233-253.

Hughes, C., Dunn, J., \& White, A. (1998). Trick or treat?: Uneven understanding of mind and emotion and executive dysfunction in "hard-to-manage" preschoolers. Journal of Child Psychology and Psychiatry, 39, 981-994.

Hughes, C., White, A., Sharpen, J., \& Dunn, J. (2000). Antisocial, angry and unsympathetic: "Hard-to-manage" preschoolers' peer problems and possible cognitive influences. Journal of Child Psychology and Psychiatry, 41, 169-179. 
Isquith, P. K., Crawford, J. S., Espy, K. A., \& Goia, G. A. (2005). Assessment of executive functioning in preschool-aged children. Mental Retardation and Developmental Disabilities Research Reviews, 11, 209-215.

Jacobs, R., Anderson, V., \& Harvey, S. (2001). Concept generation test as a measure of conceptual reasoning skills in children: Examination of developmental trends. Clinical Neuropsychological Assessment, 2, 101-117.

Jacques, S., \& Zelazo, P. D. (2001). The flexible items selection task (FIST): A measure of executive function in preschoolers. Developmental Neuropsychology, 20, 573-591.

Kalff, A. C., Hendriksen, J. G. M., Kroes, M., Vles, J. S. H., Steyaert, J., Feron, F. J. M., et al. (2002). Neurocognitive performance of 5and 6-year-old children who met the criteria for attention deficit/ hyperactivity disorder at 18 months follow-up: Results from a prospective population study. Journal of Abnormal Child Psychology, 30, 589-598.

Keenan, K., \& Shaw, D. (1997). Developmental and social influences on young girls' early problem behavior. Psychological Bulletin, $121,95-113$

Kerr, A., \& Zelazo, P. D. (2004). Development of "hot" executive function: The children's gambling task. Brain and Cognition, 55, $148-157$.

Klenberg, L., Korkman, M., \& Lathi-Nuutila, P. (2001). Differential development of attention and executive functions in 3-to-12year-old Finnish children. Developmental Neuropsychology, 20, 407-428.

Kochanska, G., Murray, K. T., \& Harlan, E. T. (2000). Effortful control in early childhood: Continuity and change, antecedents, and implications for social development. Developmental Psychology, 36, 220-232.

Korkman, M., Kemp, S. L., \& Kirk, U. (2001). Effects of age on neurocognitive measures on children ages 5-12: A crosssectional study on 800 children from the United States. Developmental Neuropsychology, 20, 331-354.

Letho, J., Juujärvi, P., Kooistra, L., \& Pulkinnen, L. (2003). Dimensions of executive functioning: Evidence from children. British Journal of Developmental Psychology, 21, 59-80.

Loeber, R., Farrington, D. P., Stouthamer-Loeber, M., \& Van Kammen, W. B. (1998). Antisocial behavior and mental health problems: Explanatory factors in childhood and adolescence. Mahwah, $\mathrm{NJ}$ : Erlbaum.

Matthys, W., Van Goozen, S. H. M., De Vries, H., Cohen-Kettenis, P. T., \& Van Engeland, H. (1998). The dominance of behavioral activation over behavioral inhibition in conduct disordered boys with or without attention deficit hyperactivity disorder. Journal of Child Psychology and Psychiatry, 39, 643-651.

Matthys, W., Van Goozen, S. H. M., Snoek, H., \& Van Engeland, H. (2004). Response perseveration and sensitivity to reward and punishment in boys with oppositional defiant disorder. European Child and Adolescent Psychiatry, 13, 362-364.

Miyake, A., Friedman, N. P., Emerson, M. J., Witzki, A. H., Howerter, A., \& Wager, T. D. (2000). The unity and diversity of executive functions and their contributions to complex "frontal lobe" tasks: A latent variable analysis. Cognitive Psychology, 41, 49-100.

Moffitt, T. E. (1993). The neuropsychology of conduct disorder. Development and Psychopathology, 5, 135-151.

Morgan, A. B., \& Lilienfeld, S. O. (2000). A meta-analytic review of the relation between antisocial behavior and neuropsychological measures of executive function. Clinical Psychology Review, 20, $113-136$.

Nigg, J. T., Hinshaw, S. P., Carte, E. T., \& Treuting, J. J. (1998). Neuropsychological correlates of childhood attention-deficit/ hyperactivity disorder: Explainable by comorbid disruptive behavior or reading problems? Journal of Abnormal Psychology, $107,468-480$.
Olson, S. L., Sameroff, A. J., Kerr, D. C. R., Lopez, N. L., \& Wellman, H. M. (2005). Developmental foundations of externalizing problems in young children: The role of effortful control. Development and Psychopathology, 17, 25-45.

Oosterlaan, J., Logan, G. D., \& Sergeant, J. A. (1998). Response inhibition in $\mathrm{AD} / \mathrm{HD}, \mathrm{CD}$, comorbid $\mathrm{AD} / \mathrm{HD}+\mathrm{CD}$, anxious and normal children: A meta-analysis of studies with the stop task. Journal of Child Psychology and Psychiatry, 39, 411-426.

Oosterlaan, J., Scheres, A., \& Sergeant, J. A. (2005). Which executive function deficits are associated with $\mathrm{AD} / \mathrm{HD}, \mathrm{ODD} / \mathrm{CD}$ and comorbid AD/HD+ODD/CD? Journal of Abnormal Child Psychology, 31(1), 69-85.

Overman, W. H. (2004). Sex differences in early childhood, adolescence, and adulthood on cognitive tasks that rely on orbital prefrontal cortex. Brain and Cognition, 55, 134-147.

Pennington, B. F. (1997). Dimensions of Executive Functions in normal and abnormal development. In N. A. Krasnegor, \& G. R. Lyon (Eds.) Development of the prefrontal cortex: Evolution, neurobiology, and behavior (pp. 265-281). Baltimore: Brooks.

Pennington, B. F., \& Ozonoff, S. (1996). Executive functions and developmental psychopathology. Journal of Child Psychology and Psychiatry, 37, 51-87.

Picton, T. W., Stuss, D. T., Alexander, M. P., Shallice, T., Binns, M. A., \& Gillingham, S. (2006). Effects of focal frontal lesions on response inhibition. Cerebral Cortex, 17, 826-838.

Sattler, J. M. (1992). Assessment of children (3rd ed.). San Diego: Jerome M. Sattler, Publisher, Inc.

Séguin, J. R., Boulerice, B., Harden, P. W., Tremblay, R. E., \& Pihl, R. O. (1999). Executive functions and physical aggression after controlling for attention deficit hyperactivity disorder, general memory and IQ. Journal of Child Psychology and Psychiatry, 40, 1197-1208.

Séguin, J. R., Nagin, D., Assaad, J., \& Tremblay, R. E. (2004). Cognitive-neuropsychological function in chronic physical aggression and hyperactivity. Journal of Abnormal Psychology, $113,603-613$.

Séguin, J. R., Pihl, R. O., Harden, P. W., Tremblay, R. E., \& Boulerice, B. (1995). Cognitive and neuropsychological characteristics of physically aggressive boys. Journal of Abnormal Psychology, 104, 614-624.

Séguin, J. R., \& Zelazo, P. D. (2005). Executive function in early physical aggression. In R. E. Tremblay, W. W. Hartup, \& J. Archer (Eds.) Developmental origins of aggression (pp. 307329). New York: Guilford.

Senn, T. E., Espy, K. A., \& Kaufmann, P. M. (2004). Using path analysis to understand executive function organization in preschool children. Developmental Neuropsychology, 26, 445-464.

Shaw, D. S., Lacourse, E., \& Nagin, D. S. (2005). Developmental trajectories of conduct problems and hyperactivity from ages 2 to 10. Journal of Child Psychology and Psychiatry, 46, 931-942.

Smidts, D. P. (2003). Development of executive processes in early childhood. PhD thesis, University of Melbourne, Melbourne, Victoria, Australia.

Smidts, D. P., Jacobs, R., \& Anderson, V. (2004). The object classification task for children (OCTC): A measure of concept generation and mental flexibility in early childhood. Developmental Neuropsychology, 26(1), 385-401.

Sonuga-Barke, E. J. S., Dalen, L., Daley, D., \& Remmington, B. (2002). Are planning, working memory, and inhibition associated with individual differences in preschool ADHD symptoms? Developmental Neuropsychology, 21, 255-272.

Speltz, M. L., DeKlyen, M., Calderon, R., Greenberg, M. T., \& Fisher, P. A. (1999). Neuropsychological characteristics and test behaviours of boys with early onset conduct problems. Journal of Abnormal Psychology, 108, 315-325.

Thorell, L. B., \& Wåhlstedt, C. (2006). Executive functioning deficits in relation to symptoms of ADHD and/or ODD in 
preschool children. Infant and Child Development, 15, 503518.

Van Goozen, S. H. M., Cohen-Kettenis, P. T., Snoek, H., Matthys, W., Swaab-Barneveld, H., \& van Engeland, H. (2004). Executive functioning in children: A comparison of hospitalised ODD and ODD/ADHD children and normal controls. Journal of Child Psychology and Psychiatry, 45(2), 284-292.

Vander Steene, G., \& Bos, A. (1997). WPPSI-R: Wechsler preschool and primary scale of intelligence (Dutch-Flemish version). Lisse: Swets \& Zeitlinger.

Webster-Stratton, C. (2001). The incredible years: Parents and children videotape series: A parenting course (BASIC). Seattle, WA: Incredible Years.
Welsh, M. C., Pennington, B. F., \& Groisser, D. B. (1991). A normative developmental study of executive function; A window of prefrontal functioning in children. Developmental Neuropsychology, 7, 131-149.

Wechsler, D. (1949). Manual for the Wechsler intelligence Scale for children. New York: The Psychological Corporation.

Wechsler, D. (1997). Wechsler preschool and primary scale of intelligence-revised (WPPSI-R). San Antonio, TX: The Psychological Corporation.

Willcutt, E. G., Doyle, A. E., Nigg, J. T., Faraone, S. V., \& Pennington, B. F. (2005). Validity of the executive function theory of attention-deficit/hyperactivity disorder: A meta-analytic review. Biological Psychiatry, 57, 1336-1346. 